\title{
On the non-periodic groups, whose subgroups of infinite special rank are transitively normal
}

\author{
L. A. Kurdachenko, I. Ya. Subbotin, and T. V. Velychko
}

\begin{abstract}
A BSTRACT. This paper devoted to the non-periodic locally generalized radical groups, whose subgroups of infinite special rank are transitively normal. We proved that if such a group $G$ includes an ascendant locally nilpotent subgroup of infinite special rank, then $G$ is abelian.
\end{abstract}

\section{Introduction}

The groups with certain prescribed properties of subgroups form one of the central subjects of research in group theory. Their investigation introduced many important concepts such as the finiteness conditions, locally nilpotence, locally solubility, subnormality, permutability, some important numerical invariants on groups, in particular, distinct group ranks, and others. Choosing specific prescribed properties and concrete families of subgroups which posses these properties, we come to distinct classes of groups. There is an enormous array of papers devoted to this theme. In the present paper we will consider the influence of the following two families of subgroups on the structure of a group. These are the family of subgroups of finite special rank and the family of transitively normal subgroups.

We say that a subgroup $H$ of a group $G$ is transitively normal if $H$ is normal in every subgroup $K \geqslant H$, in which $H$ is subnormal [1].

It is well known that the relation "to be a normal subgroup" is not transitive.

2010 MSC: Primary 20E15, 20F16; Secondary 20E25, 20E34, 20F22, 20 F50.

Key words and phrases: finite special rank, soluble group, periodic group, locally nilpotent radical, locally nilpotent residual, transitively normal subgroups. 
A group $G$ is said to be a $T$-group if this relation is transitive in $G$.

A group $G$ is said to be a $\bar{T}$-group if every subgroup of $G$ is a $T$-group.

It is not hard to see that every subgroup of $G$ is transitively normal if and only if $\mathrm{G}$ is a $\bar{T}$-group.

A group $G$ is said to be a group of a finite special rank $r$ if every finitely generated subgroup of $G$ can be generated by at most $r$ elements and there exists a finitely generated subgroup $H$ which has exactly $r$ generators [2]. The theory of groups of finite special rank is one of the best developed part of group theory ( see, for example, surveys [3-5] and the book [6] ). In the paper [7], M.R. Dixon, M.J. Evans and H. Smith initiated investigation of the groups, whose subgroups of infinite special rank have some fixed property $P$. This research has been prolonged by many authors for distinct natural properties $P$ ( see, for example, survey [5] ). In the paper [8], the study of the groups whose subgroups of infinite special rank are transitively normal was initiated. More precisely, the structure of periodic soluble groups with this property has been described there. In the current paper we begin investigation of the non-periodic groups, whose subgroups of infinite special rank are transitively normal. The main result of this paper is the following.

Theorem 1. Let $G$ be a non-periodic locally generalized radical group, whose subgroups of infinite special rank are transitively normal. If $G$ includes an ascendant locally nilpotent subgroup of infinite special rank, then $G$ is abelian.

Corollary 1. Let $G$ be a non-periodic locally generalized radical group, whose subgroups of infinite special rank are transitively normal. If $G$ includes an ascendant abelian subgroup of infinite special rank, then $G$ is abelian.

We derive the corollaries of this theorem for some types of subgroups that are transitively normal.

Recall that a subgroup $H$ is said to be contranormal in a group $G$ if its normal closure $H^{G}$ coincides with $G$.

A subgroup $H$ of a group $G$ is said to be polynormal in a group $G$ if for each subgroup $S$ including $H$ the subgroup $H$ is contranormal in $H^{S}$ [9].

It is not hard to see that every polynormal subgroup is transitively normal, so we can obtain

Corollary 2. Let $G$ be a non-periodic locally generalized radical group, whose subgroups of infinite special rank are polynormal. If $G$ includes 
an ascendant locally nilpotent subgroup of infinite special rank, then $G$ is abelian.

A subgroup $H$ is said to be paranormal in a group $G$ if $H$ is contranormal in the subgroup $\langle H, H g\rangle$ for all elements $g$ of a group $G$ [9].

Every paranormal subgroup of $G$ is polynormal in $G$ [9], so we obtain

Corollary 3. Let $G$ be a non-periodic locally generalized radical group, whose subgroups of infinite special rank are paranormal. If $G$ includes an ascendant locally nilpotent subgroup of infinite special rank, then $G$ is abelian.

A subgroup $H$ of a group $G$ is called weakly normal in $G$ if the inclusion $H^{g} \leqslant N_{G}(H)$ always implies $g \in N_{G}(H)[10] \leqslant N G(H)$

We note that every weakly normal subgroup is transitively normal, so we obtain

Corollary 4. Let $G$ be a non-periodic locally generalized radical group, whose subgroups of infinite special rank are weakly normal. If $G$ includes an ascendant locally nilpotent subgroup of infinite special rank, then $G$ is abelian.

A subgroup $H$ of a group $G$ is called weakly pronormal in $G$ if the subgroups $H$ and $H^{g}$ are conjugate in $H^{\langle g\rangle}$ for each element $g$ of the group $G[9]$.

We say that a subgroup $H$ of a group $G$ has the Frattini property if for every subgroups $K, L$ such that $H \leqslant K$ and $K$ is normal in $L$ we have $L=N_{L}(K) K$.

We note that a subgroup $H$ is weakly pronormal on $G$ if and only if $H$ has the Frattini property [9]. Clearly every subgroup having the Frattini property, is transitively normal, so we obtain

Corollary 5. Let $G$ be a non-periodic locally generalized radical group, whose subgroups of infinite special rank are weakly pronormal. If $G$ includes an ascendant locally nilpotent subgroup of infinite special rank, then $G$ is abelian.

A subgroup $H$ of a group $G$ is called pronormal in $G$ if the subgroups $H$ and $H^{g}$ are conjugate in $\left\langle H, H^{g}\right\rangle$ for each element $g$ of the group $G$. Clearly every pronormal subgroup is transitively normal, so we obtain

Corollary 6. Let $G$ be a non-periodic locally generalized radical group, whose subgroups of infinite special rank are pronormal. If $G$ includes an ascendant locally nilpotent subgroup of infinite special rank, then $G$ is abelian. 


\section{The proofs}

Lemma 1. Let $G$ be a group, whose subgroups of infinite special rank are transitively normal. Suppose that $G$ includes a normal subgroup $K$ of infinite special rank. Then $G / K$ is a $\bar{T}$-group.

Indeed, every subgroup of $G$ including $K$ has infinite special rank, so it is transitively normal in $G$. In other words, every subgroup of $G / K$ is transitively normal in $G$. It follows that $G / K$ is a $\bar{T}$-group.

Lemma 2. Let $G$ be a group, whose subgroups of infinite special rank are transitively normal.

If $H$ is a subgroup of $G$, then every subgroup of $H$ of infinite special rank is transitively normal in $H$.

If $L$ is a normal subgroup of $G$ such that $G / H$ has infinite special rank, then every subgroup of $G / L$ of infinite special rank is transitively normal in $G / L$.

If $U, V$ are subgroups of $G$ such that $U$ is normal in $V$ and $V / U$ has infinite special rank, then every subgroup of $V / U$ having infinite special rank is transitively normal in $V / U$.

The proof of this assertion is obvious.

Lemma 3. Let $G$ be a locally (soluble - by - finite) $\bar{T}$-group. Then $G$ is metabelian.

Proof. Let $u, v, x, y$ be arbitrary elements of $G$. Put $D=\langle u, v, x, y\rangle$. Then $D$ is soluble-by-finite. Let $S$ be a normal soluble subgroup of $D$ of finite index. Being a finite $\bar{T}$-group, $D / S$ must be soluble [11, Theorem $\left.1^{*}\right]$. It follows that $D$ is soluble. Notice now that a soluble $T$-group is metabelian [12, Theorem 2.3.1], so that $D$ is metabelian. In particular, $[[x, y],[u, v]]=1$. Since it is true for all elements $u, v, x, y$ of a group $G$, $[G, G]$ is abelian.

Recall that a group $G$ is said to be hyperabelian if $G$ has an ascending series of normal subgroups whose factors are abelian.

Corollary 7. Let $G$ be a hyperabelian $\bar{T}$-group. Then $G$ is metabelian.

Proof. Let

$$
\langle 1\rangle=A_{0} \leqslant A_{1} \leqslant \ldots \leqslant A_{\alpha} \leqslant A_{\alpha+1} \leqslant \ldots \leqslant A_{\gamma}=G
$$

be a series of normal subgroups whose factors are abelian. The subgroup $A_{\omega}, \omega$ is the first infinite ordinal, is locally soluble, therefore, using lemma 3 
we obtain that $A_{\omega}$ is metabelian. It follows that $A_{\omega+1}$ is soluble. Using again lemma 3 we obtain that $A_{\omega+1}$ is metabelian. Repeating these arguments and using transfinite induction we obtain that $G$ is metabelian.

Recall that a group $G$ is said to be radical if $G$ has an ascending series, whose factors are locally nilpotent. If $G$ is a radical group, then locally nilpotent radical of $G$ is non-trivial. It follows that a radical group has an ascending series of normal subgroups whose factors are locally nilpotent.

Recall that the group $G$ is called Dedekind if every its subgroup is normal. The Dedekind group $G$ has the following structure: it either is abelian, or $G=Q_{8} \times D \times P$ where $Q_{8}$ is a quaternion group of order 8, $D$ is an elementary abelian 2-group and $P$ is an abelian 2-group [13].

Corollary 8. Let $G$ be a radical $\bar{T}$-group. Then $G$ is metabelian.

Proof. Let

$\langle 1\rangle=A_{0} \leqslant A_{1} \leqslant \ldots \leqslant A_{\alpha} \leqslant A_{\alpha+1} \leqslant \ldots \leqslant A_{\gamma}=G$

be a series of normal subgroups whose factors are locally nilpotent. Clearly every locally nilpotent $\bar{T}$-group is Dedekind, in particular it is nilpotent. It follows that a radical $\bar{T}$-group is hyperabelian and we can apply Corollary 7 .

In every group $G$ the subgroup generated by its normal locally finite subgroups is locally finite. It follows that $G$ has the greatest normal locally finite subgroup. Denote this subgroup by $L f(G)$. This periodic subgroup of $G, L f(G)$, is called the locally finite radical of $G$.

A group $G$ is said to be generalized radical if $G$ has an ascending series whose factors are locally nilpotent or locally finite. If $G$ is a generalized radical group then either locally nilpotent radical of $G$ is non-trivial or locally finite radical of $G$ is non-trivial. It follows that a generalized radical group has an ascending series of normal subgroups whose factors are locally nilpotent or locally finite.

Corollary 9. Let $G$ be a generalized radical $\bar{T}$-group. Then $G$ is metabelian.

Proof. Let

$$
\langle 1\rangle=A_{0} \leqslant A_{1} \leqslant \ldots \leqslant A_{\alpha} \leqslant A_{\alpha+1} \leqslant \ldots \leqslant A_{\gamma}=G
$$

be a series of normal subgroups, whose factors are locally nilpotent or locally finite. We note that each locally finite factor of this series is soluble [11, Corollary of Theorem $\left.1^{*}\right]$. It follows that $G$ is a radical group, and we can apply Corollary 8 . 
Corollary 10. Let $G$ be a locally generalized radical $\bar{T}$-group. Then $G$ is metabelian.

Proof. Indeed, every finitely generated subgroup of $G$ is soluble by Corollary 9 , so that $G$ is locally soluble and we can apply Lemma 3 .

Corollary 11. Let $G$ be a locally generalized radical $\bar{T}$-group. If $G$ is non-periodic, then $G$ is abelian.

Proof. Since $G$ is non-periodic, it contains an element $g$ having infinite order. Let $x, y$ be the arbitrary elements of $G$. Put $D=\langle g, x, y\rangle$. Then $D$ is a generalized radical subgroup of $G$. By Corollary $10, D$ is soluble. Being infinite and finitely generated, $D$ is abelian [12, Theorem 3.3.1]. In particular, $x y=y x$. Since it is true for all elements $x, y$ of a group $G, G$ is abelian.

Lemma 4. Let $G$ be a locally generalized radical group, whose subgroups of infinite special rank are transitively normal. Suppose that $G$ includes a normal abelian torsion - free subgroup $A$ of infinite special rank. Then $G$ is abelian.

Proof. Being a subgroup of infinite special rank, $A$ includes a subgroup $B=\operatorname{Dr}_{\lambda \in \Lambda}\left\langle b_{\lambda}\right\rangle$ where each element $b_{\lambda}$ has infinite order, the set of indexes $\Lambda$ is infinite, and $\left\langle a_{\lambda}\right\rangle$ is infinite. Being infinite, $\Lambda$ includes two infinite subsets $\Gamma, \Delta$ such that $\Gamma \bigcap \Delta=\varnothing$ and $\Gamma \bigcup \Delta=\Lambda$. Then each subgroup $C=\mathbf{D r}_{\lambda \in \Gamma}\left\langle a_{\lambda}\right\rangle$ and $D=\mathbf{D r}_{\lambda \in \Delta}\left\langle a_{\lambda}\right\rangle$ has infinite special rank and, therefore, is transitively normal in $G$. The fact that $A$ is normal in $G$ implies that both subgroups $C, D$ are subnormal in $G$. Being subnormal and transitively normal in $G, C$ and $D$ are normal in $G$. The choice of $C$ and $D$ shows that $C \bigcap D=\langle 1\rangle$. Since $C$ ( respectively $D$ ) has infinite special rank, Lemma 1 implies that $G / C$ ( respectively $G / D$ ) is a $\bar{T}$ group. The choice of $C$ and $D$ shows that the factor-groups $G / C$ and $G / D$ are locally generalized radical and non-periodic. Corollary 11 of Lemma 3 implies that $G / C$ and $G / D$ are abelian. The equality $D \cap C=\langle 1\rangle$ together with Remak's Theorem implies the imbedding $G \rightarrow G / C \times G / D$, which proves that $G$ is an abelian group.

If $G$ is a group, then denote by $\operatorname{Tor}(G)$ the greatest normal periodic subgroup of $G$. $\operatorname{Tor}(G)$ is called the periodic radical of $G$. We recall that a periodic radical of a locally nilpotent group $G$ contains all elements of finite order (see, for example [6, Proposition 1.2.11] ), so that $G / \operatorname{Tor}(G)$ is a torsion-free locally nilpotent group. 
Let $G$ be a group and $\mathbf{S}$ be a family of subgroups of $G$. Then $\mathbf{S}$ is said to be a Kurosh-Chernikov series if it satisfies the following conditions:

(KC 1) $\langle 1\rangle, G \in \mathbf{S}$;

(KC 2) for each pair $A, B$ of the subgroups from $\mathbf{S}$ either is $A \leqslant B$ or $B \leqslant A$

(KC 3) for every subfamily $\mathbf{L}$ of $\mathbf{S}$ the intersection of all members of $\mathbf{L}$ belongs to $S$ and the union of all members of $\mathbf{L}$ belongs to $\mathbf{S}$; in particular, for each non-identity element $x$ of a group $G$ the union $V_{x}$ of all members of $\mathbf{S}$ not containing $x$ belongs to $\mathbf{S}$, and the intersection $\Lambda_{x}$ of all members of $\mathbf{S}$ containing $x$ belongs to $\mathbf{S}$;

(KC 4) for each non-identity element $x \in G$ the subgroup $V_{x}$ is normal in $\Lambda_{x}$.

The factor-groups $\Lambda_{x} / V_{x}$ are called the sections of the series $\mathbf{S}$.

If every subgroup of $\mathbf{S}$ is normal in $G$, then $\mathbf{S}$ is called a normal Kurosh-Chernikov series. For this case the factor-groups $\Lambda_{x} / V_{x}$ are called factors of the series $\mathbf{S}$.

Recall also that a factor $U / V$ of a group $G$ is called central if $U / V \leqslant$ $\zeta(G / V)$.

Lemma 5. Let $G$ be a locally nilpotent torsion-free group of infinite special rank. If every subgroup of $G$ of infinite special rank is transitively normal, then $G$ is abelian.

Proof. Being torsion-free and locally nilpotent, $G$ has a normal KuroshChernikov series $\mathbf{S}$, consisting of pure subgroups, whose factors are central [14]. In particular, it follows that the factors of this series are torsion-free. If $H$ is a member of $\mathbf{S}$ having infinite special rank, Lemma 1 shows that $G / H$ is a $\bar{T}$-group. Being locally nilpotent and torsion-free, $G / H$ is abelian by Lemma 3. Put

$\mathbf{L}=\{H \mid H \in \mathbf{S}$ and $H$ has infinite special rank $\}$ and $K=\bigcap \mathbf{L}$.

Since $G$ has infinite special rank, $\langle 1\rangle \in \mathbf{L}$, so that, $\mathbf{L}$ is not empty. We have seen above that for each subgroup $H \in \mathbf{L}$ the factor-group $G / H$ is abelian. Using Remak's theorem we obtain that $G / K$ is also abelian.

If $K$ has finite special rank, then there exists a positive integer $t$ such that the term of the upper central series of number $t$ includes $K$ ( it follows, for example, from Lemma 2.3.1 of the book [6] ). In this case, the group $G$ is nilpotent. Let $A$ be a maximal normal abelian subgroup of $G$. If we suppose that $A$ has finite special rank, then $G$ has finite special rank ( see, for example [6, Lemma 2.3.2] ), and we obtain a contradiction. This contradiction shows that $A$ has infinite special rank. Using now Lemma 3, we obtain that $G$ is abelian. 
Suppose now that $K$ has infinite special rank, so that $K$ is the least element of $\mathbf{L}$. Denote by $D$ the union of all members of $\mathbf{S}$ which are contained in $H$, then the factor $K / D$ is central. In particular, $G / D$ is nilpotent. If $D=\langle 1\rangle$, then $K$ is abelian and, as above, $G$ is also abelian. Therefore suppose that $D \neq\langle 1\rangle$. Let $V$ be a member of $\mathbf{S}$ such that $V \leqslant H$ and $V \neq H$. Then $V$ has finite special rank. As we have noted above, in this case there exists a positive integer $m$ such that the term of the upper central series having number $m$ includes $V$. It follows that the term $Z$ of the upper central series with the number $\omega$ ( $\omega$ is the first infinite ordinal ) includes each members of $\mathbf{S}$ which is contained in $H$. Then it includes their union $D$. The fact that $G / D$ is nilpotent implies that $G$ is hypercentral. Let $B$ be a maximal normal abelian subgroup of $G$. If we suppose that $B$ has finite special rank, then $G$ has finite special rank (see, for example [6, Lemma 2.3.2]), and we obtain a contradiction. This contradiction shows that $A$ has infinite special rank. Using now Lemma 4, we obtain that $G$ is abelian.

Repeating almost word to word the proof of Lemma 4, we can prove

Lemma 6. Let $G$ be a non-periodic locally generalized radical group, whose subgroups of infinite special rank are transitively normal. Suppose that $G$ includes a normal elementary abelian $p$ - subgroup, $p$ is a prime. Then $G$ is abelian.

Lemma 7. Let $G$ be a non-periodic locally generalized radical group, whose subgroups of infinite special rank are transitively normal. Suppose that $G$ includes a locally nilpotent normal subgroup $A=D r_{p \in \pi} A_{p}$ where $A_{p}$ is a Sylow p-subgroup of $A$ of finite special rank, $p \in \pi$, and the set $\pi$ is infinite. If $A$ has infinite special rank, then $G$ is abelian.

Proof. The fact that $A$ has infinite special rank implies that the special ranks of subgroups $A_{p}$ are not bounded. Let

$$
\pi=\left\{p_{n} \mid n \in \mathbf{N}\right\}, \pi_{k}=\left\{p_{n} \mid n>k\right\},
$$

then the subgroups $B_{k}=\mathbf{D r}_{p \in \pi k} A_{p}$ are G-invariant and have infinite special rank for all $k \in \mathbf{N}$. Then Lemma 1 shows that $G / B_{k}$ is a $\bar{T}$-group, $k \in \mathbf{N}$. Since it is not periodic, Corollary 11 of Lemma 3 implies that $G / B_{k}$ is abelian, $k \in \mathbf{N}$. Then the obvious equality $\bigcap_{k \in \mathbf{N}} B_{k}=\langle 1\rangle$ together with Remak's theorem imply that $G$ is abelian.

Corollary 12. Let $G$ be a non - periodic locally generalized radical group, whose subgroups of infinite special rank are transitively normal, and let $L$ 
be the locally nilpotent radical of $G$. If $\operatorname{Tor}(L)$ has infinite special rank, then $G$ is abelian.

Proof. Let $T=\operatorname{Tor}(L)$. Then $T=\operatorname{Dr}_{p \in \Pi(G)} T_{p}$ where $T_{p}$ is the Sylow $p$-subgroup of $T, p \in \Pi(G)$. The fact that $T$ has infinite special rank implies that either there exists a prime $q$ such that a subgroup $T_{q}$ has infinite special rank, or $T_{p}$ has finite special rank for each prime $p$ but the special ranks of subgroups $T_{p}$ are not bounded. In the second case using Lemma 7 we obtain that $G$ is abelian. Consider the first case. Then $T_{q}$ is a Dedekind group [8, Lemma 1.3]. Taking into account the structure of Dedekind group, we can see that $T_{q}$ includes a $G$-invariant infinite elementary abelian $p$-subgroup $A$. Now we can apply Lemma 6 and obtain again that $G$ is abelian.

Lemma 8. Let $G$ be a non-periodic locally generalized radical group, whose subgroups of infinite special rank are transitively normal, and let $L$ be the locally nilpotent radical of $G$. If $L$ has infinite special rank and Tor $(L)$ is a Chernikov subgroup, then $G$ is abelian.

Proof. Let $T=\operatorname{Tor}(L)$. The fact that $T$ is Chernikov implies that $T$ has finite special rank. It follows that $L / T$ has infinite special rank. Using Lemma 5 we obtain that the factor $L / T$ is abelian. It follows that $L$ is soluble. Then by Kargapolov's theorem ( see, for example [6, Corollary 9.2.20] ), $L$ includes an abelian subgroup $A$ of infinite special rank. Since $\operatorname{Tor}(A)=P$ is Chernikov, $A=P \times B$ for some subgroup $D$ ( see, for example [15, Theorem 21.2, Theorem 27.5] ). Thus $B$ is an abelian torsionfree subgroup of infinite special rank. Then $B$ is transitively normal in $G$. The fact that $T$ is a Chernikov subgroup implies that some term of upper central series of $L$ includes $T$. Since $L / T$ is abelian, $L$ is hypercentral. It follows that every subgroup of $L$ is ascendant. Being ascendant and transitively normal in $G$, the subgroup $B$ is normal [1, Lemma 1.1]. Using now Lemma 4, we obtain that $G$ is abelian.

Corollary 13. Let $G$ be a non-periodic locally generalized radical group, whose subgroups of infinite special rank are transitively normal, and let $L$ be the locally nilpotent radical of $G$. If $L$ has infinite special rank, then $G$ is abelian.

Proof. Let $T=\operatorname{Tor}(L)$. If $T$ has infinite special rank, then Corollary 12 of Lemma 7 shows that $G$ is abelian. Therefore, assume that $T$ has finite special rank. The fact that $T$ is Chernikov implies that $T$ has finite special rank. It follows that $L / T$ has infinite special rank. We have 
$T=\operatorname{Dr}_{p \in \Pi(T)} T_{p}$ where $T_{p}$ is the Sylow $p$-subgroup of $T, p \in(G)$. The fact that $T$ has finite special rank implies that $T_{p}$ is a Chernikov subgroup for each prime $p$ ( see, for example [6, Corollary 6.2.10 and Corollary 3.2.21] ). If $q$ is a prime number, then put $T_{q}=\mathbf{D r}_{p \in \Pi(T), p \neq q} T_{p}$. Then $T / T_{q}$ is a Chernikov $q$-group for each $q \in \Pi(T)$. Then Lemma 8 shows that $G / T_{q}$ is abelian. The obvious equality $\bigcap_{q \in \Pi(T)} T_{q}=\langle 1\rangle$ together with Remak's theorem imply that $G$ is abelian.

\section{Proof of the theorem}

Let $A$ be an ascendant locally nilpotent subgroup of $G$, having infinite special rank. Then the locally nilpotent radical $L$ of $G$ includes $A$ [16, Corollary of Theorem 1]. It follows that $L$ has infinite special rank. Using Corollary 13 to Lemma 8 we obtain that $G$ is abelian.

\section{References}

[1] Kurdachenko L.A., Subbotin I.Ya. Transitivity of normality and pronormal subgroups., Combinatorial group Theory, discrete groups, and number theory. Contemporary Mathematics, N.421, 2006, pp.201 - 212.

[2] Maltsev A.I. On groups of finite rank, Mat. Sbornik, N.22, 1948, pp.351-352.

[3] Dixon M.R., Kurdachenko L.A., Subbotin I.Ya. On various rank conditions in infinite groups, Algebra Discrete Math, N.4, 2007, pp.23-44.

[4] Dixon M.R., Certain rank conditions on groups, Noti di Matematica, N.2, 2008, pp. $151-175$.

[5] Dixon M.R.,Kurdachenko L.A., Pypka A.A., Subbotin I.Ya. Groups satisfying certain rank conditions, Algebra Discrete Math., N.4, 2016, pp.23-44.

[6] Dixon M.R., Kurdachenko L.A., Subbotin I.Ya. Rank of groups: the tools, characteristic and restrictions, Wiley, 2017.

[7] Dixon M.R., Evans M.J., Smith H. Locally (soluble-by-finite) groups with all proper insoluble subgroups of finite rank, Arch. Math., N.68, 1997, pp.100-109.

[8] Semko N.N., Velychko T.V. On the groups whose subgroups of infinite special rank are transitively normal, Algebra and discrete Math, N.1, 2017, pp.34-45.

[9] Ba M.S.,Borevich Z. I., On arrangement of intermediate subgroups. Rings and Linear Groups,Kubanskij Univ., Krasnodar, 1988, 14-41.

[10] Muller K.H. Schwachnormale Untergruppen: Eine gemeinsame Verallgemeinerung der normalen und normalisatorgleichen Untergruppen., Rend. Semin. Mat. Univ. Padova, N.36, 1966, pp.129-157.

[11] Robinson D.J.S. A note on finite groups in which normality is transitive., Proc. Amer. Math. Soc., N.4, 1968, pp.933-937.

[12] Robinson D.J.S. Groups in which normality is a transitive relation., Proc. Cambridge Phil. Soc., N.60, 1964, pp.21-38. 
[13] Baer R., Situation der Untergruppen und Struktur der Gruppe, S.-B. Heidelberg Akad. 2 (1933), 12-17.

[14] Glushkov V.M. On some problems of theory of torsion - free nilpotent and locally nilpotent groups, Math. Sbornik, N.1, 1952, pp.79-104.

[15] Fuchs L. Infinite abelian groups, ACADEMIC PRESS: New York, 1970.

[16] Plotkin B.I. Radical groups., Math. Sbornik, N.3, 1966, pp.507-526.

\section{CONTACT INFORMATION}

L. A. Kurdachenko, Department of Geometry and Algebra, Dnipro

T. V. Velychko National University, 72 Gagarin Av., Dnipro,

Ukraine 49010

$$
\begin{array}{r}
E \text {-Mail(s): lkurdachenko@i.ua, } \\
\text { etativ@rambler.ru }
\end{array}
$$

I. Ya. Subbotin Department of Mathematics and

NaturalSciences, National University, 5245

PacifcConcourse Drive, LA, CA 90045, USA

E-Mail(s): isubboti@nu.edu

Received by the editors: 17.03.2019. 